\title{
Atomic resolution energy-loss magnetic chiral dichroism measurements enabled by patterned apertures
}

\author{
Hasan Ali $\odot,{ }^{1}$ Devendra Negi, ${ }^{2, *}$ Tobias Warnatz $\odot,{ }^{2}$ Björgvin Hjörvarsson, ${ }^{2}$ Jan Rusz, ${ }^{2}$ and Klaus Leifer ${ }^{1, \dagger}$ \\ ${ }^{1}$ Electron Microscopy and Nano-Engineering, Applied Materials Science, Department of Engineering Sciences, Uppsala University, \\ Box 534, 75121 Uppsala, Sweden \\ ${ }^{2}$ Department of Physics and Astronomy, Uppsala University, Box 516, 75120 Uppsala, Sweden
}

(Received 31 August 2019; revised manuscript received 21 April 2020; accepted 21 April 2020; published 12 June 2020)

\begin{abstract}
Electron energy-loss magnetic chiral dichroism (EMCD) has the potential to measure magnetic properties of the materials at atomic resolution but the complex distribution of magnetic signals in the zone axis and the overlapping diffraction discs at higher beam convergence angles make the EMCD signal acquisition challenging. Recently, the use of ventilator apertures to acquire the EMCD signals with atomic resolution was proposed. Here we give the experimental demonstration of several types of ventilator apertures and obtain a clear EMCD signal at beam semiconvergence angles of $5 \mathrm{mrad}$. To simplify the experimental procedures, we propose a modified ventilator aperture which not only simplifies the complex scattering conditions but reduces the influence of lens aberrations on the EMCD signal as compared to the originally proposed ventilator apertures. In addition, this modified aperture can be used to analyze magnetic crystals with various symmetries and we demonstrate this feature by acquiring EMCD signals on different zone axis orientations of an Fe crystal. With the same aperture we obtain EMCD signals with convergence angles corresponding to atomic resolution electron probes. After the theoretical demonstration of the EMCD signal on a zone axis orientation at high beam convergence angles, this work thus overcomes the experimental and methodological hurdles and enables atomic resolution EMCD on the zone axis by using apertures.
\end{abstract}

DOI: 10.1103/PhysRevResearch.2.023330

\section{INTRODUCTION}

If one were to build an instrument for investigating spatial dependence of quantum effects at interfaces, one of the determining design criteria would be the obtained spatial resolution. The required resolution is in the range of few atomic distances to few tens of nanometers $[1,2]$ which is close to ideal for transmission electron microscope (TEM). The TEM with its analysis volume of the specimen between a few $\mathrm{nm}^{3}$ and a few $10 \mathrm{~nm}^{3}$ has been used for investigating quantum wires, quantum dots, and interfaces incorporated in a thin film or a bulk material. In the analysis of nanomagnetic materials, TEM has played a very important role since it had enabled the analysis of such materials with a resolution of below $10 \mathrm{~nm}$ using techniques of electron holography and Lorentz microscopy [3-6]. In such analysis, the resolution of the TEM has bridged the gap between the atom by atom surface analysis in the

\footnotetext{
*Present address: Stuttgart Center for Electron Microscopy, Max Planck Institute for Solid State Research, Heisenbergstrasse 1, 70569 Stuttgart, Germany.

†Klaus.Leifer@angstrom.uu.se

Published by the American Physical Society under the terms of the Creative Commons Attribution 4.0 International license. Further distribution of this work must maintain attribution to the author(s) and the published article's title, journal citation, and DOI. Funded by Bibsam.
}

scanning tunneling microscope [7] and the analysis using nonmicroscopy techniques like XMCD [8]. With recent developments in the technique of differential phase contrast (DPC) $[9,10]$ and energy-loss magnetic chiral dichroism (EMCD) [11-16], the perspective of achieving atomic resolution for the analysis of magnetic properties appears reachable. In classical EMCD experiments, two electron energy-loss spectra (EELS) are acquired at two specific scattering angles in the diffraction plane and the difference of these spectra, called the EMCD signal, is used to determine the magnetic moments of the material. One of the difficulties of the EMCD technique operating at subnanometer to atomic resolution is that high convergence angles of the focused electron probe are needed which have the drawback that the electron beam diffraction disks start to overlap in the diffraction plane making the $q$ selection needed to obtain the EMCD signal difficult. Another limitation to obtain atomic resolution EMCD is that most work hitherto was carried out having atomic planes parallel to the electron beam to reach the required diffraction geometry of a two-beam or three-beam condition [11] losing atomic column resolution. There is though one previous EMCD work carried out on a zone-axis orientation where a parallel electron beam of $50 \mathrm{~nm}$ in diameter was used in the experiments [17]. Nevertheless, Negi et al. [18] have shown in their simulations that an EMCD signal can be obtained from highly convergent electron beams and on the zone axis by using symmetric multihole apertures. To verify this result experimentally, two steps have to be taken, a first one that shows that an EMCD signal is obtained for convergences angles resulting in electron probes 
sufficient to reach atomic resolution and a second step where the developed methodology is used to obtain an atomically resolved EMCD map. Here, we have fabricated such apertures and show that from an optimization of those apertures EMCD signals can be acquired with convergence angles corresponding to electron probe sizes below $2 \AA$ enabling thus atomic resolution EMCD analysis.

In this paper, we first demonstrate EMCD signals that are obtained by the use of an eightfold ventilator aperture at a beam convergence semiangle of $\alpha=5 \mathrm{mrad}$ in a highsymmetry zone axis. We then propose and experimentally demonstrate a simplified version of the ventilator aperture which has the freedom to be used in multiple crystal symmetries keeping a zone axis orientation. Using this modified aperture we obtain a clear EMCD signal with $\alpha=10 \mathrm{mrad}$.

\section{EXPERIMENTAL DETAILS}

We used a single-crystal bcc Fe film to demonstrate the use of multihole apertures for quantitative EMCD measurements. For this purpose, a 35-nm-thick Fe film was epitaxially grown on a $\mathrm{MgO}$ (001) substrate using direct-current magnetron sputtering. The film was capped with 6-nm-thick MgO layer to prevent oxidation of the Fe layer. The TEM samples were prepared in both plan view and cross sectional geometries to carry out the EMCD experiments in a [001] and a [110] zone axis of Fe respectively. The experiments were performed on a FEI Tecnai F30 scanning TEM (STEM) operating at $300 \mathrm{kV}$ acceleration voltage equipped with a postcolumn Gatan Tridiem spectrometer. The entrance apertures of the spectrometer were replaced by the different multihole apertures described below. The TEM sample was rotated to achieve the desired rotational alignment of the diffraction pattern with respect to the fixed apertures. This was done as follows: The TEM sample was manually rotated in the sample holder outside the microscope and then inserted in the microscope. Then, a diffraction pattern (DP) was recorded and to improve the residual misalignment between the aperture and the DP, the sample holder was taken out and the sample was manually rotated again. This iterative procedure allowed for a precise alignment of the DP with respect to the aperture orientation. All the EMCD experiments were carried out in STEM mode. The beam semiconvergence angle was set to $5 \mathrm{mrad}$ for the EMCD experiments carried out under the [001] zone axis, For the [110] zone axis, semiconvergence angles of 5, 7.5, and $10 \mathrm{mrad}$ were used to obtain the EMCD signals. The inner and outer collection angles were set to 12.5 and $25 \mathrm{mrad}$ in all the cases which are the optimum values predicted from theory. In all the cases, a focused electron probe was scanned across the sample and the EELS spectra were acquired in the form of two-dimensional (2D) EELS images at each scan point. The EELS edges in the 2D images were aligned using cross correlation to get rid of residual aberrations of the spectrometer. The chiral plus $(C+)$ and chiral minus $(C-)$ EELS spectra were extracted by drawing line profiles in the 2D EELS images. The background of the EELS spectra was subtracted by using a power-law model and the postedge of the resulting spectra was normalized [19]. In the EMCD results presented in this paper, the intensity of the EELS spectra was normalized to maximum 1 by dividing both the $C+$ and $C-$ spectra with
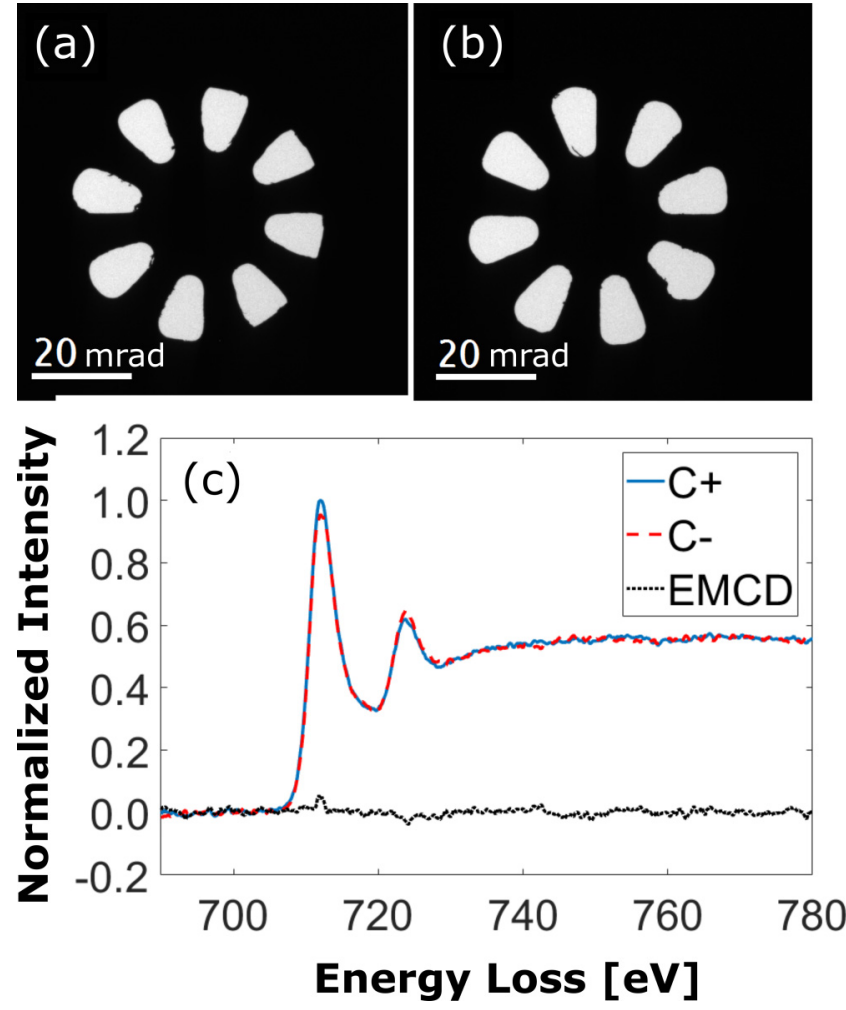

FIG. 1. (a), (b) CCD images of two ventilator apertures fixed in the spectrometer entrance apertures. The beam semiconvergence angle was $5 \mathrm{mrad}$. A 2D EELS image was acquired at each scan point with an acquisition time of $5 \mathrm{~s} / \mathrm{scan}$ point. For each line scan, an EELS spectrum was obtained by integrating the 2D data of 15 scan points and taking the intensity profile along the spectral trace. (c) The postprocessed EELS spectra for the two apertures along with their difference (EMCD) signal are shown.

the highest number of counts at $L_{3}$ edge of the $C+$ spectrum. This intensity normalization does not affect the measured $m_{\mathrm{L}} / m_{\mathrm{S}}$ values but visually helps the reader to estimate the EMCD signal strength on a relatively simple scale.

\section{RESULTS AND DISCUSSION}

To verify the simulations shown in Ref. [18], we designed two single signal eight-hole (SS8) apertures, also called ventilator apertures, for the [001] zone axis (ZA) of bcc Fe. This ventilator aperture can acquire one component (positive or negative) of magnetic signal in one orientation and either the sample or the aperture needs to be rotated to acquire the other component of the signal. This operation of rotating the sample or the aperture can lead to difficulties in maintaining the same experimental conditions between the two acquisitions. We solved this problem by building two ventilator apertures side by side in the spectrometer aperture holder. The two apertures were rotated by $22.5^{\circ}$ with respect to each other in a way that they could acquire opposite magnetic signals for a fixed orientation of the diffraction pattern as shown in Figs. 1(a) and 1(b). The acquisition of two magnetic signals can now be accomplished by scanning a sample area twice just switching the apertures in between the acquisitions. The TEM sample was oriented on the [001] ZA and an area of the sample 


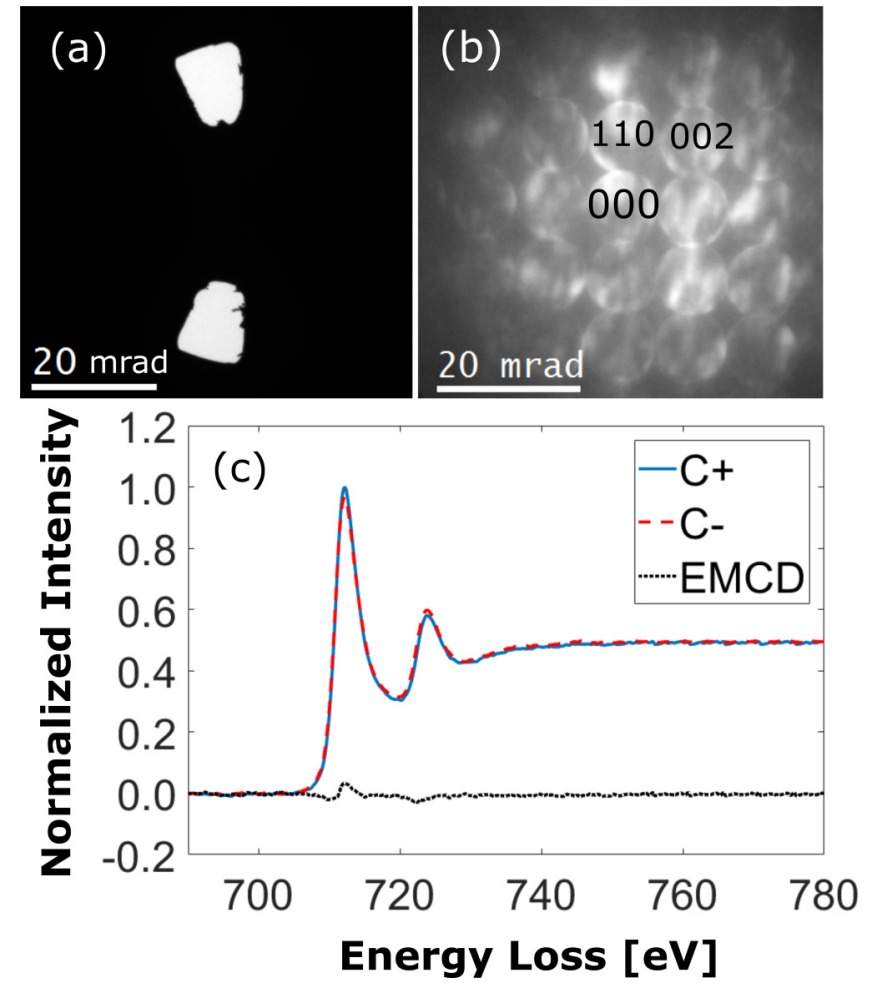

FIG. 2. (a) CCD image of the DS2 aperture (b) an electrondiffraction pattern from the region of measurement. In the STEM mode, the electron beam with a semiconvergence angle of $5 \mathrm{mrad}$ was scanned in a line across the sample and a 2D EELS image was acquired at each scan point. The acquisition time was $5 \mathrm{~s} / \mathrm{scan}$ point. Data of 60 scan points was integrated to generate a final 2D EELS image containing two spectral traces for each aperture. The EELS spectra were extracted by taking the intensity profiles along each spectral trace. (c) The postprocessed EELS spectra and the obtained EMCD signal are shown.

was chosen for the measurements where the sample thickness was approximately $35 \mathrm{~nm}$. Spectral data in the form of line scans were acquired using each of the apertures from the same region of the sample. The EELS spectra were extracted and processed in the way described in the experimental section. As compared to the $C+$ spectrum, the $C$ - spectrum has a weaker $\mathrm{Fe} L_{3}$ edge and a stronger $\mathrm{Fe} L_{2}$ edge signal as shown in Fig. 1(c). This change in edge intensity is equally shown in the difference spectrum.

The ventilator apertures shown in Fig. 1 can detect the EMCD signals in ZA geometry, but their use for the acquisition of EMCD signals has a few limitations. One drawback is the complexity of the apertures themselves. The physics of scattering, and even more, the physics of inelastic electron scattering is complex for these apertures and it is nontrivial to compare the experimental results with the simulations. We think therefore that the use of higher convergence angles for these apertures needs a careful and stepwise experimental methodology, starting experiments with one- or two-hole apertures to first understand the influence of every single hole aperture on the EMCD signal. A difficulty from an experimental point of view is that the EELS spectrometer is built originally for round apertures. Here, for the ven-
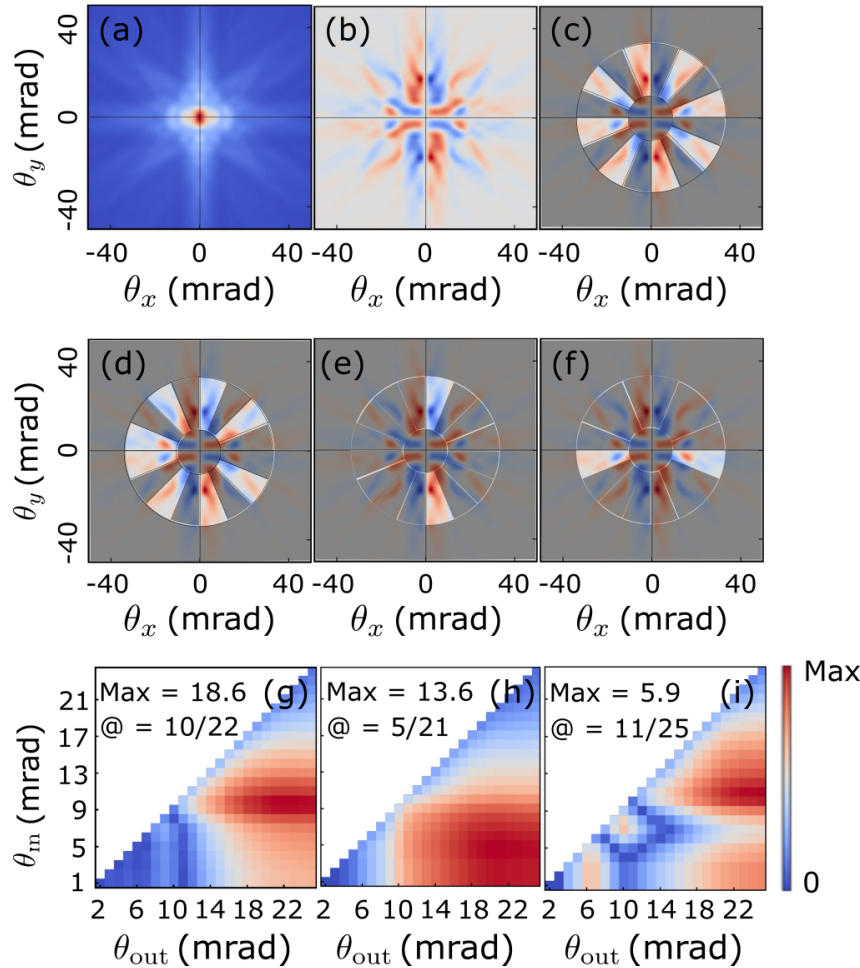

FIG. 3. Simulations of the nonmagnetic (a) and magnetic (b) component of the inelastic-scattering cross section at the $L_{3}$ edge of iron. Beam of $5 \mathrm{mrad}$ convergence semiangle accelerated by $300 \mathrm{kV}$ is passing parallel to [110] zone axis of 20-nm-thick iron crystal. In (c) and (d) the ventilator and the DS7 apertures are superposed over the magnetic signal shown in (b). In (e) and (f) the DS2 aperture is superposed over the magnetic signal with aperture holes parallel to (100) and (110) axes respectively. Panels (g)-(i) show the optimum inner/outer collection angles calculated for the ventilator and the DS2 apertures shown in (d)-(f) respectively.

tilator apertures, in principle, each individual hole has its own spectrometer transmission function. In the alignment process of the spectrometer, it is thus very hard to align the spectrometer for these apertures with many holes located in different directions with respect to the spectrometer axis. The resulting measurements might thus include some artifacts coming from the nonideal spectrometer alignment. Another disadvantage of the ventilator aperture is that it can only be used for one specific crystal symmetry and a new aperture needs to be designed for every different crystal symmetry as well as for every zone axis of interest of the same crystal. Here we propose and have built a simplified form of the ventilator aperture which can be used to get EMCD measurements in multiple crystal symmetries and is much easier to align in the spectrometer. We call this aperture the double signal two-hole (DS2) aperture. The additional advantage of the DS2 aperture is that it can acquire both positive and negative magnetic signals simultaneously. In our recent work we discussed the advantages of simultaneous acquisition of the two EELS spectra for EMCD measurements [20]. We experimentally show that the DS2 aperture allows the detection of EMCD signals with beam convergence angles high enough to enable atomic resolution. To explore the behavior of 

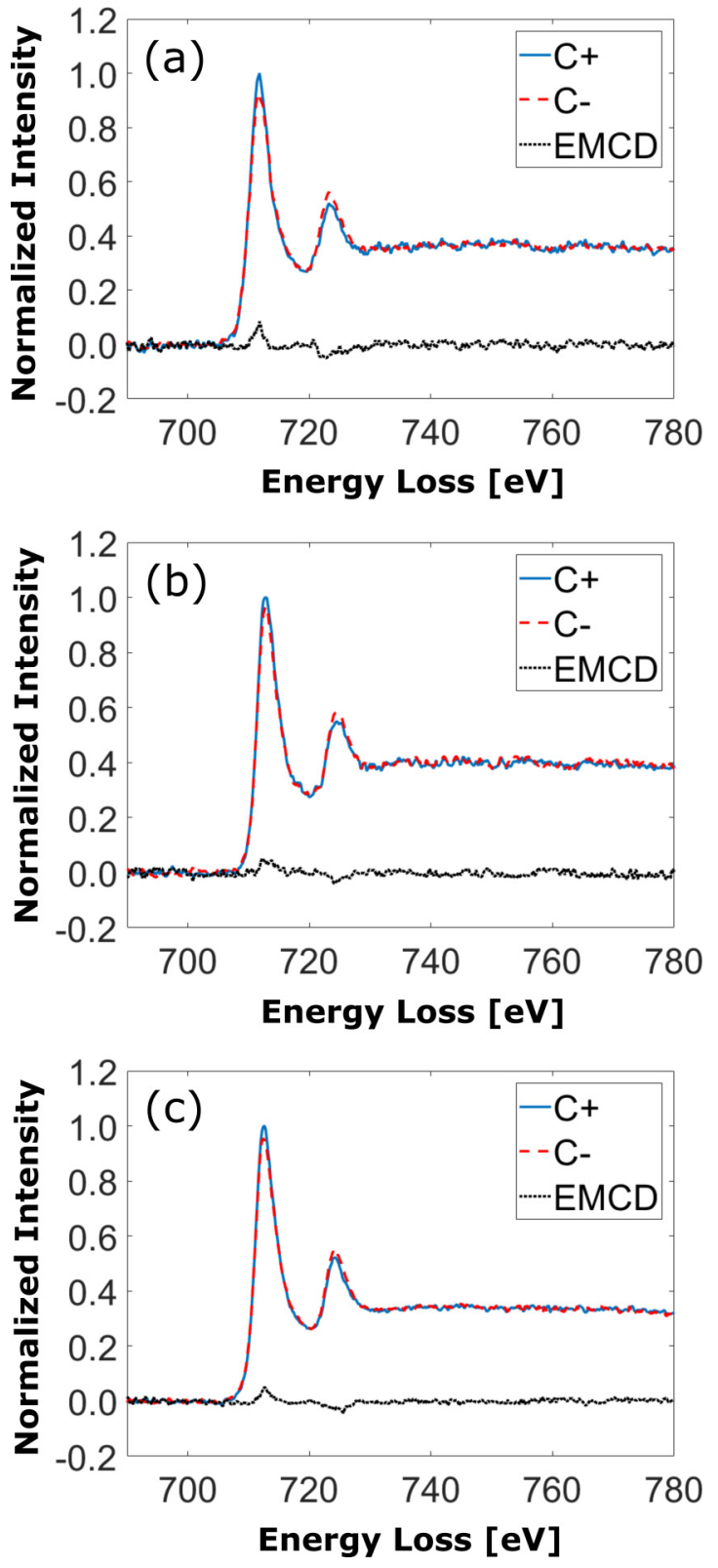

FIG. 4. EMCD signals acquired using DS2 aperture for beam semiconvergence angles of (a) 5, (b) 7.5, and (c) $10 \mathrm{mrad}$ are shown. In STEM mode, the electron beam with different convergence angles was line scanned across the Fe film and a 2D EELS image was acquired at each scan point. The acquisition time for (a) and (b) was $3 \mathrm{~s} /$ scan points and $2 \mathrm{~s} / \mathrm{scan}$ point for (c). The final EELS spectra were obtained by integrating 30 scan points for (a) and (b) and 50 scan points for (c) The plus and minus EELS spectra were extracted from the spectral traces for the upper and lower holes of the aperture respectively.

the DS2 aperture for different crystal symmetries, we applied it for EMCD measurements in two different zone axes [001] and [110] of bcc Fe. For the measurements on the [001] ZA, the same sample and similar experimental conditions were used as described above for the ventilator aperture. Figure 2 shows the charge-coupled device (CCD) image of the DS2 aperture, the electron-diffraction pattern acquired in the region of measurement, and the resulting EMCD signal. One of the
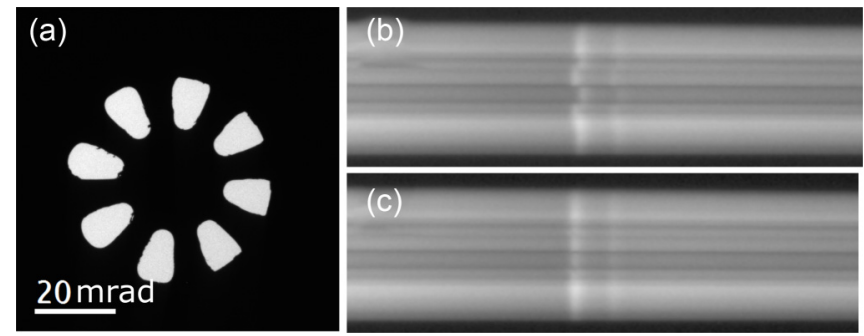

FIG. 5. (a) CCD image of the SS8 aperture. (b) Raw spectral data acquired using the SS8 aperture; residual aberrations can be seen in the form of zigzag of the EELS edges. (c) Corrected spectra data after cross correlating the peaks.

concerns about the DS2 aperture could be that the signal strength or the signal-to-noise ratio would be lower than the ventilator aperture but this can be compensated by increasing the dwell time/scan point or integrating more scan points in the map.

To apply the DS2 aperture in [110] ZA we have performed simulations of the inelastic electron scattering [21] of an electron beam with semiconvergence angle of $5 \mathrm{mrad}$, accelerated by $300 \mathrm{kV}$, on a 20-nm-thick crystal of bcc iron. In calculations presented here, the beam direction was set along the [110] zone axis. Results are summarized in Fig. 3 . Note that for zone axis [110] the symmetry of the diffraction pattern is lower, when compared to the [001] zone axis treated in Ref. [18]. Reduced symmetry of the diffraction pattern is clearly visible from both nonmagnetic [Fig. 3(a)] and magnetic [Fig. 3(b)] components of the inelastic scattering cross section at the iron $L_{3}$ edge. Superposing the eighthole ventilator aperture over the magnetic signal distribution [Fig. 3(c)] shows that not all holes collect a magnetic signal of the same sign. In fact some holes collect an approximately even amount of positive and negative EMCD. This led us to design the DS2 aperture shape, which should be equally efficient for both [110] and [001] zone axes.

According to the simulations for [110] ZA, the DS2 aperture can be aligned either parallel to the (100) or (110) axis in the diffraction plane as shown in Figs. 3(e) and 3(f). The latter orientation was chosen as the aperture stays further away from the high intensity first-order Bragg reflections which may disturb the very weak EMCD signal. We used the same collection angles as in the previous experiments. A cross-sectional $\mathrm{Fe}$ sample was used for the measurements in [110] ZA. The sample thickness was approximately $25 \mathrm{~nm}$ in the area of measurements. The experiment was performed for three semiconvergence angles 5, 7.5, and $10 \mathrm{mrad}$ of the incident electron probe. The obtained EMCD signals are shown in Fig. 4.

It is worth noting that a clear EMCD signal is visible on both the $L_{3}$ and $L_{2}$ edges in all three cases given in Fig. 4. At a semiconvergence angle of $10 \mathrm{mrad}$, the diffraction limited electron probe has a diameter of $1.2 \AA$ which is smaller than the lattice plane spacings in many magnetic materials. Thus, in an aberration corrected microscope, the setup should be capable to obtain atomically resolved EMCD maps.

In the above EMCD experiments using the ventilator apertures, a 2D EELS image was acquired at each beam position of 

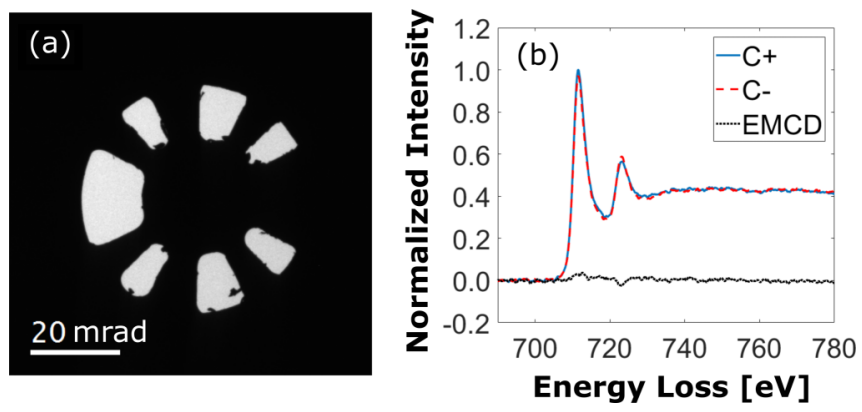

FIG. 6. (a) CCD image of DS7 aperture. The same experimental conditions were used as mentioned above for the ventilator apertures. The plus and minus EELS spectra were extracted from the spectral trace produced by the biggest aperture hole in the center. (b) Postprocessed EELS spectra along with the EMCD signal are shown.

a line scan instead of acquiring directly the EELS spectrum as performed in conventional STEM-EELS spectrum imaging. The acquisition of 2D EELS images provides a possibility to correct some of the residual aberrations of the EELS spectrometer in postprocessing steps. It seems important to highlight this aspect since due to the complex noncircular shape of the apertures, the alignment of the spectrometer is not straightforward and would need to be optimized further. We have used a MATLAB script to correct the apparent aberrations in the spectra.

Moreover, this kind of dataset gives the freedom to extract the EELS spectra from optimum scattering angles within the aperture range to minimize artifacts from high intensity diffraction spots on the EMCD signal (see Fig. 5). In fact we also optimized the design of the double signal sevenhole (DS7) aperture proposed in Ref. [18]. We cut aperture holes now smaller in order to avoid intensity of crystalline reflections to enter the aperture [Fig. 6(a)]. The outer part of the spectral trace does not seem to be well focused with this aperture, probably due to the nonsymmetric shape of the aperture. Indeed, when evaluating the inner part of the spectral trace which corresponds to the electron intensity transmitted through the biggest hole in the DS7 aperture and which now is not transmitting intensity from strong Bragg reflections, we obtain a clear EMCD signal [Fig. 6(b)]. This indicates that filtering out high intensity crystalline reflections from the aperture holes improves the strength of the EMCD signal.

To check the quality and precision of the data, we have applied sum rules [22,23] to all of the EMCD signals shown above and obtained the $m_{\mathrm{L}} / m_{\mathrm{S}}$ values for each of them which are shown in Table I. All values of orbital to spin magnetic
TABLE I. $m_{\mathrm{L}} / m_{\mathrm{S}}$ values calculated for EMCD signals acquired with different apertures.

\begin{tabular}{ll}
\hline \hline Aperture type & $m_{\mathrm{L}} / m_{\mathrm{S}}$ values \\
\hline Ventilator $([001] \mathrm{ZA}, \alpha=5 \mathrm{mrad})$ & $0.050 \pm 0.018$ \\
DS2 ([001] ZA, $\alpha=5 \mathrm{mrad})$ & $0.041 \pm 0.010$ \\
DS2 ([110] ZA, $\alpha=5 \mathrm{mrad})$ & $0.045 \pm 0.024$ \\
DS2 ([110] ZA, $\alpha=7.5 \mathrm{mrad})$ & $0.062 \pm 0.020$ \\
DS2 ([110] ZA, $\alpha=10 \mathrm{mrad})$ & $0.045 \pm 0.014$ \\
DS7 ([001] ZA, $\alpha=5 \mathrm{mrad})$ & $0.090 \pm 0.011$ \\
\hline \hline
\end{tabular}

ratio are in the $0.04-0.09$ range which is close to the values determined by other techniques $[24,25]$ and EMCD measurements $[12,26]$ for bcc Fe. The $m_{\mathrm{L}} / m_{\mathrm{S}}$ values obtained for the ventilator and the DS2 aperture (both [001] and [110] ZAs) are quite similar within the error bars. Only the $m_{\mathrm{L}} / m_{\mathrm{S}}$ values obtained for DS7 aperture are higher than the expected values. In the experiments, we found it difficult to correct aberrations of the spectrometer for DS7 aperture as compared to the two other apertures and we believe that this is one of the reasons for the overestimated $m_{\mathrm{L}} / m_{\mathrm{S}}$ values.

\section{CONCLUSIONS}

We have used three different types of apertures to acquire EELS spectra on zone axis orientations of monocrystalline $\mathrm{Fe}$ in order to extract EMCD signals. The acquisition strategy has been optimized for those apertures. From all apertures, EMCD signals are obtained on both $L_{3}$ and $L_{2}$ EELS edges and obtained $m_{\mathrm{L}} / m_{\mathrm{S}}$ ratios are in good agreement with previous works. Using a double signal two-hole aperture to simultaneously acquire the two conjugate EELS spectra enabled us to obtain an EMCD signal at a semiconvergence angle of $10 \mathrm{mrad}$, which corresponds to an electron probe size of $1.2 \AA$. Thus, this work enables atomic scale resolved EMCD measurements for convergent electron beams by the use of apertures and on a zone-axis orientation.

\section{ACKNOWLEDGMENTS}

We acknowledge the contributions of S. Sanz in fabricating and optimizing the Fe films. We acknowledge E. Lindholm for his great availability to fabricate this large variety of apertures. We gratefully acknowledge the generous support from the Swedish Science Council, VR Grants No. 201605259 and No. C0367901, the support from the Knut and Alice Wallenberg Stiftelse and the Swedish Foundation for Internationalization of Cooperation in Research and Higher Education, STINT, Grant No. IG2009 2017.
[1] T.-W. Kim, C.-H. Cho, B.-H. Kim, and S.-J. Park, Appl. Phys. Lett. 88, 123102 (2006).

[2] S. Koohfar, A. B. Georgescu, A. N. Penn, J. M. LeBeau, E. Arenholz, and D. P. Kumah, npj Quantum Mater. 4, 25 (2019) .

[3] C. Phatak, A. K. Petford-Long, and M. De Graef, Curr. Opin. Solid State Mater. Sci. 20, 107 (2016).
[4] A. Tonomura, Ultramicroscopy 47, 419 (1992).

[5] H. Lichte and M. Lehmann, Adv. Imaging Electron Phys. 123, 225 (2002).

[6] R. E. Dunin-Borkowski, T. Kasama, A. Wei, S. L. Tripp, M. J. Hytch, E. Snoeck, R. J. Harrison, and A. Putnis, Microsc. Res. Tech. 64, 390 (2004). 
[7] Q. H. Wu and J. Y. Kang, Mater. Manuf. Process. 22, 22 (2007).

[8] T. Funk, A. Deb, S. J. George, H. Wang, and S. P. Cramer, Coord. Chem. Rev. 249, 3 (2005).

[9] J. N. Chapman, P. E. Batson, E. M. Waddell, and R. P. Ferrier, Ultramicroscopy 3, 203 (1978).

[10] A. Edstrom, A. Lubk, and J. Rusz, Phys. Rev. B 99, 174428 (2019).

[11] P. Schattschneider, S. Rubino, C. Hébert, J. Rusz, J. Kuneš, P. Novák, E. Carlino, M. Fabrizioli, G. Panaccione, and G. Rossi, Nature (London) 441, 486 (2006).

[12] H. Lidbaum, J. Rusz, A. Liebig, B. Hjörvarsson, P. M. Oppeneer, E. Coronel, O. Eriksson, and K. Leifer, Phys. Rev. Lett. 102, 037201 (2009).

[13] T. Thersleff, J. Rusz, B. Hjörvarsson, and K. Leifer, Phys. Rev. B 94, 134430 (2016)

[14] Z. Wang et al., Nat. Mater. 17, 221 (2018).

[15] J. Rusz, S. Muto, J. Spiegelberg, R. Adam, K. Tatsumi, D. E. Bürgler, P. M. Oppeneer, and C. M. Schneider, Nat. Commun. 7, 12672 (2016).

[16] J. C. Idrobo, J. Rusz, J. Spiegelberg, M. A. McGuire, C. T. Symons, R. R. Vatsavai, C. Cantoni, and A. R. Lupini, Adv. Struct. Chem. Imag. 2, 5 (2016) .
[17] D. Song, J. Rusz, J. Cai, and J. Zhu, Ultramicroscopy 169, 44 (2016).

[18] D. Negi, J. Spiegelberg, S. Muto, T. Thersleff, M. Ohtsuka, L. Schönström, K. Tatsumi, and J. Rusz, Phys. Rev. Lett. 122, 037201 (2019).

[19] H. Lidbaum, J. N. Rusz, S. Rubino, A. Liebig, B. H. Orvarsson, P. M. Oppeneer, O. Eriksson, and K. Leifer, Ultramicroscopy 110, 1380 (2010).

[20] H. Ali, T. Warnatz, L. Xie, B. Hjörvarsson, and K. Leifer, Ultramicroscopy 196, 192 (2019).

[21] J. Rusz, Ultramicroscopy 177, 20 (2017).

[22] L. Calmels, F. Houdellier, B. Warot-Fonrose, C. Gatel, M. J. Hytch, V. Serin, and E. Snoeck, Phys. Rev. B 76, 060409 (2007).

[23] J. Rusz, O. Eriksson, P. Novak, and P. M. Oppeneer, Phys. Rev. B 76, 060408 (2007).

[24] C. T. Chen, Y. U. Idzerda, H. J. Lin, N. V. Smith, G. Meigs, E. Chaban, G. H. Ho, E. Pellegrin, and F. Sette, Phys. Rev. Lett. 75, 152 (1995).

[25] M. B. Stearns, in 1.1.2.7 Form Factors, Densities and Magnetic Moments, datasheet from Landolt-Börnstein Group III: Condensed Matter, Vol. 19A: (Springer-Verlag, Berlin, 1986), p. 52.

[26] B. Warot-Fonrose, C. Gatel, L. Calmels, V. Serin, and P. Schattschneider, Ultramicroscopy 110, 1033 (2010). 